\title{
Virtual intracranial electroencephalography for epilepsy surgery using ictal magnetoencephalography
}

\section{Miao Cao}

University of Melbourne

Daniel Galvis

University of Birmingham

\section{Simon Vogrin}

University of Melbourne

William Woods

Swinburne University of Technology

\section{Sara Vogrin}

University of Melbourne

\section{Fan Wang}

Chinese Academy of Sciences

\section{Wessel Woldman}

University of Birmingham

John Terry

University of Birmingham

\section{Andre Peterson}

University of Melbourne

Chris Plummer ( $\nabla$ chris.plummer@svha.org.au )

Swinburne University of Technology https://orcid.org/0000-0001-5434-1343

\section{Mark Cook}

University of Melbourne

\section{Article}

Keywords: Virtual Intracranial Electroencephalography, Dynamical Network Models, Virtual Resection, Magnetoencephalography, Source Imaging

Posted Date: February 2nd, 2021

DOl: https://doi.org/10.21203/rs.3.rs-153655/v1 
License: (c) (i) This work is licensed under a Creative Commons Attribution 4.0 International License. Read Full License

Version of Record: A version of this preprint was published at Nature Communications on February 22nd, 2022. See the published version at https://doi.org/10.1038/s41467-022-28640-x. 


\section{Abstract}

Modelling the interactions that arise from neural dynamics in seizure genesis is challenging but important in the effort to improve the success of epilepsy surgery. Dynamical network models developed from physiological evidence offer insights into rapidly evolving brain networks in the epileptic seizure. A major limitation of previous studies in this field is the dependence on invasive cortical recordings with constrained spatial sampling of brain regions that might be involved in seizure dynamics. Here, we propose a novel approach, virtual intracranial electroencephalography (ViEEG), that combines noninvasive ictal magnetoencephalographic imaging (MEG), dynamical network models and a virtual resection technique. In this proof-of-concept study, we show that ViEEG signals reconstructed from MEG alone preserve critical temporospatial characteristics for dynamical approaches to identify brain areas involved in seizure generation. Our findings demonstrate the advantages of non-invasive ViEEG over the current presurgical 'gold-standard' - intracranial electroencephalography (iEEG). Our approach promises to optimise the surgical strategy for patients with complex refractory focal epilepsy.

\section{Introduction}

Modelling complex systems like the brain is challenging ${ }^{1}$, particularly when studying time-evolving interactions that arise from normal and aberrant neural dynamics across multiple temporal and spatial scales ${ }^{2,3,4}$. Patterns of brain activation and interactions form the neural correlates of brain states and behaviour, which are fundamental to understanding the underlying mechanisms of brain function ${ }^{5}$. Developing and validating mathematical and computational models of brain function and neural dynamics has been a key mission for neuroscience research in the last few decades.

Dynamical network models provide great capacity to probe the underlying mechanisms of complex neural dynamics. Inspired by early studies of excitatory and inhibitory neurons, investigators have developed dynamical models of neural mass and neural mass networks, which connect an ensemble of neural mass models into macroscopic neural systems ${ }^{1,6,7,8,9}$. Employing dynamical models, multiple attempts have been made to understand the mechanisms underlying normal ${ }^{10,11}$ and pathological neural dynamics $^{12,13,14}$. Dynamical network models have also been applied to neurophysiological data recorded from the human brain to develop specific hypotheses towards clinical application ${ }^{15,16,17,18}$.

A major limitation of previous studies in this field is the dependence on invasive intracranial electrode recordings of cortical activity when applying dynamical network models to experimental data. This can lead to insufficient sampling of brain networks with biased representation of the actual systems involved ${ }^{19}$. Moreover, invasive cortical recordings are relatively costly to obtain from animal models and only possible to obtain from the diseased human brain, as in epilepsy surgery planning, with inherent risks ${ }^{20}, 21$. Non-invasive neuroimaging, such as high density electroencephalography (HDEEG) and magnetoencephalography (MEG), offer high temporal resolution and whole brain spatial coverage ${ }^{22}$. Unlike functional magnetic resonance imaging (fMRI), electromagnetic fields recorded over the head 
surface by EEG and MEG represent the linear summation of collective source activity ${ }^{23}$. Advances in EEG and MEG source imaging techniques have improved the capacity to project the recorded surface electromagnetic fields back to source activity with high spatial resolution when sufficient signal-to-noise ratios are obtained ${ }^{23,24}$.

Epilepsy affects about $1 \%$ of the global population and at least one third of epilepsy patients have seizures that are refractory to medication ${ }^{25,26}$. While surgery can be an effective treatment for pharmacorefractory epilepsy, it is widely underused ${ }^{27}$. Presurgical evaluation is particularly challenging when MRI shows no clear lesion, a large complex lesion, or multiple potential epileptogenic lesions ${ }^{28,29}$. Such cases stand to benefit from non-invasive dynamical approaches to better characterise the brain networks involved in seizure dynamics. Here, we aim to apply a dynamical network approach to source reconstructed non-invasive ictal MEG data and we introduce the novel concept of virtual intracranial EEG (ViEEG). A set of sources, or virtual electrodes, ${ }^{30}$ are defined in the reconstructed individualised MRI brain where ictal source signals can be reconstructed as ViEEG signals. We then construct patient-specific functional networks that are integrated in dynamical network models to determine the effects of the network structure on seizure transitions. Specifically, brain regions responsible for seizure generation are identified by evaluating the contribution of each individual node to the network excitability, i.e., the likelihood of a particular node affecting the transition to a seizure. From this evaluation, we attempt to non-invasively define a virtual ictogenic zone (VIZ) and assess our ViEEG derived dynamical network models against the preoperative HDEEG and MEG ictal source localisation (ESL, MSL), the clinical intracranial EEG (iEEG) localisation, and the epileptogenic zone (EZ) as defined by resection linked to long term postoperative outcome. In doing this, we aim to address several questions. Can we reconstruct ictal ViEEG signals that have distinct spatial and temporal characteristics of epileptiform discharges? If so, can dynamical network models using ictal ViEEG signals guide a surgical strategy that characterises the EZ while identifying brain areas that are less likely to be involved in the EZ? Finally, can dynamical network models using ictal ViEEG signals provide unique information to the current clinical localisation, including the iEEG seizure onset zone (SOZ) and HDEEG and MEG source localisation, in characterising the EZ?

\section{Materials And Methods}

\section{Dataset and Ethics}

The study was based on the dataset in Plummer et $\mathrm{al}^{31}$. Subjects' consent was obtained according to the Declaration of Helsinki. Ethics approval for the research protocol was given by The Human Research Ethics Committees of St Vincent's Hospital and Swinburne University of Technology, Melbourne. Thirteen patients (seven males, six females, age range 10-54 years, median 33 years; disease duration 3-32 years) had drug-resistant focal epilepsy with either no visible MRI lesion (10 patients) or a complex lesion (3 patients). Each patient had an hour-long simultaneous HDEEG (ANT Waveguard® 72-94 electrode cap including 12-electrode inferior temporal array, 10-10 positions) acquired on one of two EEG amplifier 
systems (ANT ASAlab ${ }^{\circledR}$, Enschede; Compumedics SynampRT ${ }^{\circledR}$ ) and MEG (Elekta Triux ${ }^{\circledR} 306$ sensors, 102 magnetometers, 204 planar gradiometers), sampled at $1000 \mathrm{~Hz}$ or $5000 \mathrm{~Hz}$ with anti-aliasing filter set at $330 \mathrm{~Hz}$ or $1650 \mathrm{~Hz}$ respectively. Bad channels for all MEG data were checked prior to applying temporal extension to signal source separation (tSSS) using Maxfilter® v2.2.10-15 (Elekta Oy, Helsinki) for interference suppression (correlation limit 0.98 and sliding window of 10 seconds). Independent onesecond interval clock triggers acquired on each system were used to synchronise HDEEG and MEG offline, verified by comparing ECG channel signal phase from each independent modality. MEG head coils, HDEEG electrode positions, and PAN (pre-auricular, nasion) co-ordinates were digitised (Polhemus Fastrak $\left.{ }^{\circledR}\right)$ in common space for MRI co-registration; digitised points were cross-validated with optical sensor tracking (NDI Polaris Vicra $\left.{ }^{\circledR}\right)$. Applying a second-order bandpass Hann FFT filter $(1-100 \mathrm{~Hz})$, seizures were independently marked by a neurologist (C.P.) and a clinical scientist (S.V.) using Curry $7 \AA$ (Compumedics Neuroscan ${ }^{\circledR}$, Hamburg). A total of 36 ictal MEG events were captured from 12 patients (interictal-only activity in one MRI-normal patient) (Table 1). Pre-operative MPRage MRI was acquired as part of clinical investigation as well as post-operative $\mathrm{CT}$ to confirm resection margins. A post-iEEG implantation CT scan confirmed implantation locations in the seven patients who had iEEG grids or strips - Patient 7 had an intraoperative depth electrode and four patients (Patients 2, 3,6,8) went straight to surgery based on non-invasive data. As raw iEEG recordings were not available to us for two external patients (only iEEG reports for Patients 7,9) we were able to compare ViEEG to iEEG results in six patients (Patients $1,4,5,10,11,12$ ). Based on findings from Plummer et al ${ }^{31}$, ictal early-MSL preceded early-ESL in six patients (Patients $1,3,4,5,10,11$ ), while ictal early-ESL preceded early-MSL in five patients (Patients 2, 6, 8, 9, 12) Ictal discharges were not localisable in one patient (Patient 7) (Table 1). Using standardised low resolution tomographic analysis (sLORETA), Plummer et $\mathrm{al}^{31}$ found there is usually a lead-lag relationship between early-MSL and early-ESL for a given averaged ictal discharge and it was the earliest solution (defined as the first occurring early-MSL or early-ESL solution from discharge take-off that explained $90 \%$ of the signal variance) that was the best predictor of the EZ.

\section{Virtual iEEG}

We propose a novel concept (ViEEG) that consists of multiple virtual electrodes or virtual sensors guided by HDEEG and MEG source imaging ${ }^{30} 32$. An example of ViEEG defined for a patient is given in Fig. 1. ViEEG was defined using similar electrode configurations to iEEG, such as grid, strip and depth arrays, and a uniform spacing $(10 \mathrm{~mm})$ between virtual electrodes to allow a direct comparison between iEEG and non-invasive ViEEG. In this study, ViEEG was defined for each patient solely using information from $M L^{31}$ (not ESL), while blinded to other clinical information. Thus, the locations of ViEEG electrodes extensively covered MSL of averaged ictal discharges (early-, mid-upstroke and late-peak phase solutions $)^{31}$. ViEEG comprising over 500 virtual electrodes is less computationally feasible for dynamical modelling and so we did not define ViEEG to cover the whole brain in this study. We used Curry $8 \AA$ (Compumedics Neuroscan ${ }^{\circledR}$, Hamburg) software and pre-operative MRI scans to generate realistic boundary element method models with a single layer, i.e., inner skull surface ${ }^{33}$. Ictal ViEEG signals were reconstructed using a linearly constrained minimum variance beamformer technique ${ }^{34}$. Technical details 
of MEG signal processing and source reconstruction are presented in Supplementary material (ViEEG signal reconstruction). A total of 25 from 36 seizures could be reconstructed using ViEEG with distinct morphological and spatial characteristics from background activity (Supplementary Table 5). Eleven seizures reconstructed from the ictal MEG data did not present identifiable morphological features of epileptiform discharges and hence were excluded from analysis. ViEEG configurations are presented in Supplementary Figs. 5-16.

\section{Blinded Analysis}

To ensure the validity of ViEEG and dynamical network models, M.C. was blinded to the surgical outcomes, pathology, iEEG findings, and ESL solutions; M.C. had no knowledge of which solution (earlyESL or early-MSL) was the earliest solution for each patient when defining ViEEG. All other team members were blinded to the locations and configurations of ViEEG. When analysing and modelling ictal ViEEG signals, D.G. was blinded to clinical information, including resection margins, iEEG findings, source localisation and surgical outcomes, and ViEEG locations and configurations. Only time-series of ictal ViEEG signals (no information on ViEEG locations, resection margin, pathology or surgical outcomes) were given to construct functional networks and apply dynamical network models. Patient information was de-identified and patient numbers were randomised from the previous publication ${ }^{31}$. Clinical information and ViEEG configurations were only unblinded to D.G., W.W. and J.T. after results from network models were finalised.

\section{Dynamical Network Models and Virtual Resection}

Functional networks were constructed from ictal ViEEG signals using two connectivity methods, amplitude envelope correlation (AEC) and mutual information (MI) ${ }^{17,35,36,37,38}$. Both methods do not conservatively correct field leakage effects in reconstructed source signals ${ }^{36}$. Similar to connectivity analysis of iEEG data, we treated each ViEEG electrode as a node and connectivity between a pair of nodes expressed by the edge-strength. A surrogate correction threshold was then applied to functional networks to preserve edges that were statistically significant ${ }^{37}$. Next, a generative dynamical network model was integrated to each node, simulating the change of network excitability when a node was virtually resected from the network ${ }^{17,39}$. Specifically, we calculated the change in time that the network spends in the seizure state over the total simulation time after a node is removed from the network structure. The effect of the removal of a node is quantified by the so-called Node Ictogenicity (NI), where a positive $\mathrm{NI}$ indicates the removal of that node effected a decrease in the seizure-likelihood. This model has been presented previously $17,40,41,42$. The set of nodes that have significantly large (Mann-WhitneyWilcoxon U-test and Bonferroni-Holms correction survival) and positive $\mathrm{NI}$ values relative to other nodes is defined as the Virtual Ictogenic Zone (VIZ). Any nodes outside the VIZ are defined as non-ictogenic nodes. For each MEG seizure, our approach identified two VIZs (AEC-VIZ and MI-VIZ) from AEC and MI constructed networks. Each VIZ also has two aspects, 'hotspot' and 'boundary'. We defined a VIZ hotspot as nodes in the top $20 \% \mathrm{NI}$ values among all VIZ nodes and boundary as the outer line of the VIZ. The VIZ hotspot (or top NI value nodes) has been shown to better predict the EZ, while the VIZ boundary can 
capture the entirety of the EZ and identify non-ictogenic brain areas that are less likely to overlap with the $\mathrm{EZ}^{17,41,42}$. See also Supplementary material (Network model).

\section{Statistical Analysis}

A mixed-effects logistic regression model was employed to evaluate the statistical significance of nodallevel (virtual electrode) concordance between a VIZ (hotspot and boundary) and clinical localisation (resection margin, iEEG SOZ and HDEEG-MEG source localisation) based on odds ratios (OR) with 95\% confidence intervals $(\mathrm{Cl})$. Akaike Information Criterion (AIC) $)^{43}$ and Bayesian Information Criterion (BIC $)^{44}$ were reported. We also calculated the precision and recall of respective boundaries and hotspots of AEC$\mathrm{VIZ}$ and $\mathrm{MI-VIZ}$ in predicting the resection margin and the earliest source localisation solution when seizures are grouped based on long-term (at least 24-month follow-up) surgical outcomes (seizure-free and non-seizure-free. F-scores, the harmonic mean of precision of VIZ hotspot and recall of VIZ boundary, were reported. See also Supplementary material (Statistical analysis).

\section{Data Availability}

All data are available upon request.

\section{Results}

Twelve patients with 25 seizures captured by simultaneously acquired HDEEG and MEG were included in our analysis (Table 1, Supplementary Table 5).

\section{Clinical features of ictal ViEEG signals}

The first question we addressed is whether we can reconstruct ictal ViEEG signals with distinct clinical characteristics to enable dynamical network models to identify brain areas that are involved and not involved in increased in silico seizure likelihood. In Fig. 2, we demonstrate a side-by-side comparison of ViEEG and iEEG signals from Patient 5. Ictal waveforms from ViEEG can be visually identified solely from sources in anterior hippocampal and basal temporal structures, which is consistent with the iEEG SOZ. Representative ictal ViEEG signals of 25 seizures are shown in Supplementary Figs. 5-16.

\section{$\mathrm{VIZ}$ hotspot and boundary characterise the EZ and predict the clinical localisation}

The next question we asked is whether the VIZ can devise a strategy that characterises the EZ with sufficient precision and recall. Successful prediction of the EZ by the VIZ hotspot is demonstrated in Fig. 2. Although the VIZ boundary is much more extensive than the $E Z$, it captures the entirety of the $E Z$ and also identifies non-ictogenic brain areas. The dynamical network models (using two different network inference techniques, AEC-VIZ and MI-VIZ) obtain high recall in predicting the EZ (Supplementary Fig. 4 for seizure-free group). Mixed-effects logistic regression models suggest AEC-VIZ and MI-VIZ significantly predict the resection margin at nodal level (Fig. 6A). F-scores (harmonic mean of precision of VIZ hotspot and recall of $\mathrm{VIZ}$ boundary) for $\mathrm{MI}-\mathrm{VIZ}$ (median=0.75) in predicting the $\mathrm{EZ}$ (i.e., resection margin for 
seizure-free patients) suggest our approach helps delineate the EZ and identify non-ictogenic brain regions based on recall of MI-VIZ (Fig. 6B, Supplementary Fig. 4). The spatial overlap between the VIZ and clinical localisation are demonstrated on a per patient and per seizure basis in Supplementary Table 3 and Supplementary Table 4. Furthermore, we find AEC-VIZ and MI-VIZ hotspots and boundaries predict the iEEG SOZ and the early-MSL solution (Fig. 6A, Supplementary Table 1, Supplementary Table 2). AECVIZ and MI-VIZ hotspots and boundaries are less likely to predict mid-MSL and late-MSL solutions (Fig. 6A, Supplementary Table 1, Supplementary Table 2), particularly when mid-MSL and late-MSL do not overlap with the corresponding early-MSL solution - Patient 2 (Supplementary Fig. 6), Patient 6 (Supplementary Fig 10), Patient 11 (Fig. 3, Supplementary Fig 15), Supplementary Fig 16).

\section{MI-VIZ predicts the optimal source localisation solution}

The earliest solution (the first-occurring early-MSL or early-ESL SLORETA solution) has been reported as the best predictor of the $\mathrm{EZ}^{31}$. In this study, we are interested in whether VIZ identified by dynamical network models predicts the earliest solution and not just the early-MSL. AEC-VIZ and MI-VIZ significantly predict the earliest solution (Fig. 6A, Supplementary Table 1 and Supplementary Table 2). Specifically, we find that the MI-VIZ predicts the early-ESL instead of early-MSL in three patients - Patient 6 (Fig. 4, Supplementary Fig. 10), Patient 8 (Supplementary Fig. 12), and Patient 12 seizure 1 (Fig. 5, Supplementary Fig. 16) - out of the total five patients who have early-ESL as the earliest solution. This is also demonstrated in Fig. 6A as higher ORs are seen for MI-VIZ hotspot and boundary prediction of the earliest solution against the early-MSL alone (also see Supplementary Table 1 and Supplementary Table 2).

\section{MI-VIZ predicts the EZ and clinical localisation better than AEC-VIZ}

AIC and BIC were calculated from mixed-effects logistic regression models evaluating a statistical association between $\mathrm{AEC}-\mathrm{VIZ}$ and $\mathrm{MI}-\mathrm{VIZ}$ and the resection margin as well as the clinical localisation (Supplementary Table 1, Supplementary Table 2). Lower AIC and BIC values suggest higher predictive power for the model. As shown in Supplementary Table 2, the MI-VIZ boundary consistently has lower AIC and $\mathrm{BIC}$ values than the $\mathrm{AEC}-\mathrm{VIZ}$ boundary in predicting the resection margin and clinical localisation. Further, the MI-VIZ hotspot has lower AIC and BIC values than corresponding AEC-VIZ hotspot values in predicting the resection, the earliest solution, early-MSL and late-MSL solutions, while AEC-VIZ hotspots better predict the iEEG SOZ and mid-MSL solution than MI-VIZ hotspots (Supplementary Table 1). Examples can be seen in Patient 6 (Fig. 4, Supplementary Fig. 10) and Patient 12 seizure 1 (Fig. 5, Supplementary Fig. 16), where MI-VIZ hotspots predict the resection margin and the earliest solution, while AEC-VIZ hotspots do not.

\section{Discussion}

In this study we used a multi-disciplinary approach to objectively characterise the ictal brain networks from non-invasive MEG data without the risks and constraints of iEEG. We have demonstrated that non- 
invasive ictal ViEEG signals preserve the most important characteristics for spatial distribution and morphology. Dynamical network models and a virtual resection technique were applied to ictal ViEEG signals to identify a sub-network VIZ that helps elucidate the EZ. Alternative surgical strategies can potentially be devised from VIZ results for non-seizure-free patients. More crucially, the VIZ from MEG data alone can predict the earliest solution out of MSL and ESL, the earliest solution best informing the likely EZ in our recent study using simultaneous HDEEG and MEG data ${ }^{31}$.

To our knowledge, this is the first study that reconstructs ictal source signals using a high number of spontaneous seizures captured by $\mathrm{MEG}^{31}$. Ictal ViEEG signals from at least one seizure per patient present distinct characteristics of ictal events, such as hyper-synchronised rhythms, clear transitions from background activity to a seizure state, and spatial patterns of seizure propagation. Such characteristics of ictal ViEEG are also confirmed by ictal iEEG data from the same patient (Fig. 2). These findings also support previous studies that show epileptic activity from deep structures can be detected and reconstructed using MEG ${ }^{45}$, 46 . From the 36 seizures recorded by MEG, 11 seizures do not present identifiable morphological features of ictal activity and hence were not included in the analysis. We suspect that compared to source localisation, source reconstruction may require higher SNRs to resolve identifiable ictal features in source space. As well, certain geometries of anatomical structures, such as gyral areas, may impair MEG source reconstruction accuracy. These 11 seizures might be amenable to source reconstruction with corresponding ictal HDEEG signals (the subject of our future work in a larger cohort). Ictal source signals have been reconstructed using ictal scalp EEG in prior studies $47,48,49$. However, as opposed to MEG, scalp EEG signals are more distorted when the electrical field propagates through inhomogeneous head tissue. More sophisticated techniques are needed to process and analyse the EEG signals in source space ${ }^{49}$.

This study demonstrates proof-of-concept that dynamical network models using ViEEG signals identify a sub-network VIZ that provide a valid characterisation of the EZ and prediction of the clinical localisation. This finding is significant for the following reasons. First, it demonstrates the feasibility of translating dynamical network models developed from iEEG to non-invasive ViEEG. Second, our proposed approach also identifies non-ictogenic brain areas that are less likely to overlap with the $E Z$, which may help clinicians fine-tune clinical hypotheses to be tested using invasive approaches. Third, our approach is data-driven and requires less human input compared to routine clinical investigations, making it a more objective assessment. As opposed to the original work of Goodfellow et al ${ }^{17}$ that only analysed the first seizure from each patient, we analysed all seizures that were successfully reconstructed from ictal MEG data. This lends confidence to our approach, as consistency of VIZ hotspots was found between seizures for a given patient - refer to Patient 2 (Supplementary Fig. 6), Patient 8 (Supplementary Fig. 12), and Patient 11 (Supplementary Fig. 15).

Ding et $\mathrm{al}^{47}$ is the first study to reconstruct ictal EEG source signals to localise the EZ in source space and investigated the causal interaction patterns to identify the primary epileptic sources. Recent work from Lopes et $\mathrm{al}^{41}$ and Sohrabpour et al ${ }^{49}$ applied network models and connectivity analysis to EEG source- 
space networks and demonstrated the feasibility of non-invasive lateralisation and characterisation of the EZ. Motivated by recent studies using invasive iEEG data ${ }^{17,18,50,51}$, our work extends the dynamical network models and virtual resection from Goodfellow et al ${ }^{17}$ and Lopes et al ${ }^{41}$ to ictal MEG source signals, which has achieved similar performance in characterising the EZ as Sohrabpour et al ${ }^{49}$ but with a different imaging modality. It is also notable that we analysed the MEG data recorded from complex cases only (MRI-normal or complex lesions) using a ViEEG approach that is intuitive to clinicians given its resemblance to iEEG arrays. Future work is needed to compare different techniques using EEG and MEG source signals.

The iEEG SOZ is often regarded as a subset of the $\mathrm{EZ}^{52}$. This is in part because resection is often performed beyond the extent of the iEEG SOZ and includes non-SOZ electrodes to ensure the removal of the entire putative EZ. However, the resection margin between $\mathrm{SOZ}$ and non-SOZ electrodes is often determined by the experience of the treating team which is less objective and less amenable to hypothesis-testing. Recent network studies ${ }^{17,50}$ indicate there may exist a regulatory mechanism surrounding the $\mathrm{SOZ}$ in the form of pathological dynamics that synchronise and de-synchronise the network and hence regulate seizure generation and propagation. Such regulatory mechanisms merit further consideration in the surgical work-up ${ }^{50}$. Our VIZ, particularly the hotspots, can non-invasively offer such an objective boundary for surgical planning. Further, ViEEG combined with dynamical network models enables hypothesis-testing to assess surgical strategies prior to resection by virtually resecting one or more nodes from the network ${ }^{17,42,51,53}$. For example, all non-seizure-free post-operative patients revealed pre-operative VIZ hotspots that sat outside the resection margins - Patient 1 (Supplementary Fig. 5), Patient 3 (Supplementary Fig. 7), Patient 4 (Supplementary Fig. 8), Patient 9 (Supplementary Fig. 13), Patient 10 (Supplementary Fig. 14), and Patient 11 (Fig. 3, Supplementary Fig. 15).

There has been a long-standing discussion on network models versus source localisation for epilepsy and epilepsy surgery ${ }^{54,55}$. An important question that has been raised is whether network models using EEG and MEG reconstructed source data better characterise the EZ than the solutions offered by source localisation. As demonstrated by Sohrabpour et al ${ }^{49}$, connectivity imaging provides similar accuracy to source localisation in determining the extent of the EZ using sophisticated source imaging algorithms on ictal HDEEG data. Our simultaneously acquired HDEEG-MEG dataset and prospective study validating the clinical utility of electromagnetic source localisation offers the unique opportunity to address such a question ${ }^{31}$. To the best of our knowledge, this is the first study that comprehensively compares findings from dynamical network modelling of ictal MEG data with source localisation solutions using simultaneous HDEEG and MEG. In our previous work, the earliest sLORETA solution of early-ESL and early-MSL best characterises the EZ than either ESL, MSL, or combined electromagnetic source localisation (EMSL) alone ${ }^{31}$. On combining ictal ViEEG signals from MEG alone with dynamical network models, MI-VIZ predicts the earliest solution that can be only offered by source localisation with two modalities (HDEEG and MEG data). Specifically, MI-VIZ can predict the earliest solution better than predicting the early-MSL solution (Fig. 6A). This finding suggests dynamical network models can provide valuable information beyond source localisation using a single modality. 
We also observe that our proposed approach identifies the wider extent of the VIZ to the EZ as well as inter-seizure variability. The extent of the VIZ is likely to reflect propagation of seizure activity ${ }^{56}$. Our previous work argues that source localisation of ictal discharges at the mid to late phase of the averaged discharge complex usually reflects areas involved in seizure propagation. Statistical analysis suggests AEC-VIZ and MI-VIZ hotspot and boundary are less likely to predict mid-MSL and late-MSL (Fig. 6A, Supplementary Table 1 and Supplementary Table 2). Hence, the VIZ is perhaps not a simple representation of seizure propagation networks but its extent could still be affected by seizure propagation. More detailed analysis of seizure propagation networks using iEEG seizures is needed to test this hypothesis.

Two connectivity methods - linear and nonlinear coupling between signals - were employed to better understand the underlying network structures responsible for seizure generation. AEC is believed to solely characterise linear correlations between amplitude envelopes ${ }^{57,58}$, while MI measures both linear and nonlinear relationships by quantifying shared and unique information between two time-series ${ }^{59}$. Our results demonstrate that $\mathrm{MI}-\mathrm{VIZ}$ outperforms $\mathrm{AEC}-\mathrm{VIZ}$ in characterising the $\mathrm{EZ}$ and predicting the clinical localisation, which suggests a connectivity approach that captures both linear and non-linear interactions might offer more information about ictal network structures than an approach that only captures linear interactions. This finding also lends support to a previous theoretical model using nonlinear dynamics from the seizure onset zone to identify the EZ from seizure propagation and predict seizure propagation and termination ${ }^{16,60}$. More broadly, our evidence supports the view that ictogenesis involves complex network neural systems that are highly nonlinear across multiple temporo-spatial scales ${ }^{60}$.

We find six non-seizure-free patients have at least one VIZ hotspot node outside the resection margin Patient 1 (Supplementary Fig. 5), Patient 3 (Supplementary Fig. 7), Patient 4 (Supplementary Fig. 8), Patient 9 (Supplementary Fig. 13), Patient 10 (Supplementary Fig. 14), and Patient 11 (Fig. 3, Supplementary Fig. 15) - which has been previously reported using ictal iEEG data ${ }^{17}$. An example is given in Patient 11 (Fig. 3, Supplementary Fig. 15), where both VIZ hotspot and iEEG SOZ are superior to the resection margin and this patient is non-seizure-free with a $60 \%$ seizure reduction rate (Engel III). Our results suggest alternative surgical strategies can be devised for non-seizure-free patients with, perhaps, reduced need for invasive monitoring for those patients who are being considered for a second resection. Some VIZs also overlap eloquent cortex as occurs in Patient 10 (Supplementary Fig. 14) and Patient 11 (Supplementary Fig. 15), which is not accounted for in our proposed approach. The balance between seizure outcome and compromise of eloquent cortical function may be integrated into future models to better facilitate decision-making for patients and clinicians.

There are some limitations in this study. Only ictal data were analysed. This is because our dynamical network models investigate the change of cortical excitability that are more likely to be revealed during a seizure state. Our model is phenomenological and does not include details of physiology that underlies the functioning of individual epilepsy patients. Findings from our proposed approach can only serve as a statistical biomarker that offers complementary clinical information to the pre-surgical evaluation. We did 
not employ connectivity methods that reduce spurious connections (often introduced by volume conduction) in source space to construct functional networks because in our study we looked at dynamical network models to characterise the EZ for epilepsy surgery, instead of investigating neural mechanisms. More details on volume conduction in source-space networks are discussed in the Supplementary material (Volume Conduction and functional networks). In addition, the computational workload does not allow us to model all the sources (5000-25000 sources) at once from the whole brain at a spatial resolution $(10 \mathrm{~mm})$ comparable to $\mathrm{iEEG}$ electrodes. At present, the feasible scale of networks is between 100 and 300 nodes for dynamical modelling and results to be interpreted. In a prospective fashion, ViEEG with on feasible network scale can be moved around freely and modelled iteratively across the whole-brain, although admittedly the ideal scenario is to model a ViEEG covering the wholebrain at once (refer to Considerations for ViEEG locations in Supplementary material). Our study is retrospective with a modest number of patients and seizures analysed compared to studies using iEEG seizures, although it contains one of the highest seizure counts obtained across ictal MEG studies. Our findings motivate further investigation in methodology and clinical utility using multi-centre datasets or prospective studies to devise and optimise surgical strategies objectively and safely.

\section{Conclusions}

We have used non-invasively acquired ictal MEG data to reconstruct ViEEG signals with distinctive morphological and spatial characteristics that are comparable to patient-specific invasively acquired ictal iEEG data. VIZ identified by dynamical network models using two connectivity methods (AEC and MI) can predict the resection margin and iEEG SOZ; they also devise an alternative surgical strategy for patients with suboptimal post-operative seizure control. Importantly, VIZ using ictal MEG data alone can predict optimal source localisation solutions that were previously derived from simultaneous HDEEG and MEG data. Our findings suggest ViEEG combined with dynamical network models can provide valuable information beyond source localisation using single modality ictal MEG, thus offering unique clinical utility in the pre-surgical evaluation of complex drug refractory epilepsy cases.

\section{Abbreviations}

MEG = magnetoencephalography, $\mathrm{iEEG}=$ intracranial electroencephalography, $\mathrm{ViEEG}=$ virtual intracranial electroencephalography , EZ = epileptogenic zone, $S O Z$ = seizure onset zone, HDEEG = high density electroencephalography, $\mathrm{VIZ}=$ virtual ictogenic zone, $M S L=$ MEG source localisation, $E S L=$ HDEEG source localisation, $\mathrm{AEC}=$ amplitude envelope correlation, $\mathrm{MI}=$ mutual information, $\mathrm{AEC}-\mathrm{VIZ}=$ virtual ictogenic zone using amplitude envelope correlation, $\mathrm{MI}-\mathrm{VIZ}=$ virtual ictogenic zone using mutual information, $\mathrm{NI}=$ node ictogenicity

\section{Declarations}

\section{Acknowledgements}


The authors acknowledge the facilities, and the scientific and technical assistance of the National Imaging Facility at the Swinburne Node, Swinburne University of Technology with particular thanks to $\mathrm{Dr}$ Rachel Batty, Ms Mahla Cameron-Bradley, and Ms Johanna Stephens for their technical support. We acknowledge the Australian National Imaging Facility for the support of W. Woods and the MEG system at Swinburne University of Technology. We acknowledge the neurologists Dr Simon Harvey (Royal Children's Hospital Melbourne), A/Prof John Archer (Austin Hospital Melbourne), A/Prof Wendyl D'Souza (St Vincent's Hospital Melbourne), A/Prof Ross Carne (St Vincent's Hospital Melbourne), and the neurosurgeons A/Prof. Michael Murphy (St Vincent's Hospital Melbourne), Mr. Kristian Bulluss (St Vincent's Hospital Melbourne), and Ms Wirginia Maixner (Royal Children's Hospital Melbourne) whose patients were included in the study. The authors would also like to thank Prof. Thomas Knosche, Dr. Christian Rummel and Dr. Marinho Lopes for their insightful discussions on MEG source imaging and dynamical models.

\section{Funding}

M.C. acknowledges the support from Australian Government Research Training Scholarship and St Vincent's Health Foundation, Australia. M.J.C., A.P and J.R.T. acknowledge the financial support from the Royal Society International Exchanges Award (grant number IE170112). D.G. and J.R.T. acknowledge the generous support of the Wellcome Trust Institutional Strategic Support Award (grant no.

204909/Z/16/Z). W.W. acknowledges the financial support of the MRC via grant (MR/N01524X/1). J.R.T acknowledges the financial support of the EPSRC via grant (EP/N014391/1). F.W. acknowledges the support from Youth Innovation Promotion Association at the Chinese Academy of Sciences via grant (2019096).

\section{Author contributions}

Study design: M.C., S.V., W.W., A.P., C.P., M.J.C; Data Acquisition: M.C., S.V., C.P.; Analysis: M.C., D.G., S.V., W.W., S.V., F.W., W.W., J.T., A.P., C.P., M.J.C.; Writing original draft: M.C., C.P.; Writing review and editing: M.C., D.G., S.V., W.W., S.V., F.W., W.W., J.T., A.P., C.P., M.J.C; Supervision: J.T., A.P., C.P., M.J.C.

\section{Competing interests}

Authors declare no competing interests.

\section{Supplementary material}

This section contains additional information on the following: Network model, Features of ictal ViEEG signals, Considerations for ViEEG locations, ViEEG signal reconstruction, Volume conduction and functional networks, Different thresholds to define hotspot of VIZ, Statistical analysis, with Supplementary figures and tables.

\section{References}


1. Breakspear M, Terry JR, Friston KJ. Modulation of excitatory synaptic coupling facilitates synchronization and complex dynamics in a biophysical model of neuronal dynamics. Network 14, 703-732 (2003).

2. Breakspear M, Stam CJ. Dynamics of a neural system with a multiscale architecture. Philos Trans $R$ Soc Lond B Biol Sci 360, 1051-1074 (2005).

3. Stam CJ. Modern network science of neurological disorders. Nat Rev Neurosci 15, 683-695 (2014).

4. Fornito A, Zalesky A, Breakspear M. The connectomics of brain disorders. Nat Rev Neurosci 16, 159172 (2015).

5. Tong F, Pratte MS. Decoding patterns of human brain activity. Annu Rev Psycho/ 63, 483-509 (2012).

6. Jansen BH, Rit VG. Electroencephalogram and visual evoked potential generation in a mathematical model of coupled cortical columns. Biol Cybern 73, 357-366 (1995).

7. Lopes da Silva FH, Hoeks A, Smits H, Zetterberg LH. Model of brain rhythmic activity. The alpharhythm of the thalamus. Kybernetik 15, 27-37 (1974).

8. Wendling F, Bellanger JJ, Bartolomei F, Chauvel P. Relevance of nonlinear lumped-parameter models in the analysis of depth-EEG epileptic signals. Biol Cybern 83, 367-378(2000).

9. David O, Friston KJ. A neural mass model for MEG/EEG: coupling and neuronal dynamics. Neuroimage 20, 1743-1755 (2003).

10. Robinson PA, Rennie CJ, Rowe DL. Dynamics of large-scale brain activity in normal arousal states and epileptic seizures. Phys Rev E Stat Nonlin Soft Matter Phys 65, 041924 (2002).

11. Freyer F, Roberts JA, Becker R, Robinson PA, Ritter P, Breakspear M. Biophysical mechanisms of multistability in resting-state cortical rhythms. J Neurosci 31, 6353-6361 (2011).

12. Wendling F, Bartolomei F, Bellanger JJ, Chauvel P. Epileptic fast activity can be explained by a model of impaired GABAergic dendritic inhibition. Eur J Neurosci 15, 1499-1508 (2002).

13. Breakspear M, Roberts JA, Terry JR, Rodrigues S, Mahant N, Robinson PA. A unifying explanation of primary generalized seizures through nonlinear brain modeling and bifurcation analysis. Cereb Cortex 16, 1296-1313 (2006).

14. Terry JR, Benjamin O, Richardson MP. Seizure generation: the role of nodes and networks. Epilepsia 53, e166-169 (2012).

15. Jirsa VK, et al. The Virtual Epileptic Patient: Individualized whole-brain models of epilepsy spread. Neuroimage 145, 377-388 (2017). 
16. Proix T, Bartolomei F, Guye M, Jirsa VK. Individual brain structure and modelling predict seizure propagation. Brain 140, 641-654 (2017).

17. Goodfellow M, Rummel C, Abela E, Richardson MP, Schindler K, Terry JR. Estimation of brain network ictogenicity predicts outcome from epilepsy surgery. Sci Rep 6, 29215 (2016).

18. Sinha $\mathrm{N}$, et al. Predicting neurosurgical outcomes in focal epilepsy patients using computational modelling. Brain 140, 319-332 (2017).

19. Parvizi J, Kastner S. Promises and limitations of human intracranial electroencephalography. Nat Neurosci 21, 474-483 (2018).

20. Nagahama $Y$, et al. Intracranial EEG for seizure focus localization: evolving techniques, outcomes, complications, and utility of combining surface and depth electrodes. J Neurosurg, 1-13 (2018).

21. Platt M, Sperling MR. A comparison of surgical and medical costs for refractory epilepsy. Epilepsia 43 Suppl 4, 25-31 (2002).

22. Lopes da Silva F. EEG and MEG: relevance to neuroscience. Neuron 80, 1112-1128 (2013).

23. He K, et al. A high-performance compact magnetic shield for optically pumped magnetometer-based magnetoencephalography. Rev Sci Instrum 90, 064102 (2019).

24. Little S, Bonaiuto J, Meyer SS, Lopez J, Bestmann S, Barnes G. Quantifying the performance of MEG source reconstruction using resting state data. Neuroimage 181, 453-460 (2018).

25. Kwan P, Brodie MJ. Early identification of refractory epilepsy. N Engl J Med 342, 314-319 (2000).

26. Thurman DJ, et al. Standards for epidemiologic studies and surveillance of epilepsy. Epilepsia $\mathbf{5 2}$ Suppl 7, 2-26 (2011).

27. Vakharia VN, Duncan JS, Witt JA, Elger CE, Staba R, Engel J, Jr. Getting the best outcomes from epilepsy surgery. Ann Neurol 83, 676-690 (2018).

28. Nowell M, Miserocchi A, McEvoy AW, Duncan JS. Advances in epilepsy surgery. J Neurol Neurosurg Psychiatry 85, 1273-1279 (2014).

29. Duncan JS, Winston GP, Koepp MJ, Ourselin S. Brain imaging in the assessment for epilepsy surgery. Lancet Neurol 15, 420-433 (2016).

30. Brookes MJ, et al. A general linear model for MEG beamformer imaging. Neuroimage 23, 936-946 (2004).

31. Plummer C, Vogrin SJ, Woods WP, Murphy MA, Cook MJ, Liley DTJ. Interictal and ictal source localization for epilepsy surgery using high-density EEG with MEG: a prospective long-term study. 
Brain 142, 932-951 (2019).

32. Hillebrand A, Singh KD, Holliday IE, Furlong PL, Barnes GR. A new approach to neuroimaging with magnetoencephalography. Hum Brain Mapp 25, 199-211 (2005).

33. Fuchs M, Drenckhahn R, Wischmann HA, Wagner M. An improved boundary element method for realistic volume-conductor modeling. leee Transactions on Biomedical Engineering 45, 980-997 (1998).

34. Van Veen BD, van Drongelen W, Yuchtman M, Suzuki A. Localization of brain electrical activity via linearly constrained minimum variance spatial filtering. IEEE Trans Biomed Eng 44, 867-880 (1997).

35. Kraskov A, Stogbauer $\mathrm{H}$, Grassberger P. Estimating mutual information. Phys Rev E Stat Nonlin Soft Matter Phys 69, 066138 (2004).

36. Palva JM, et al. Ghost interactions in MEG/EEG source space: A note of caution on inter-areal coupling measures. Neuroimage 173, 632-643 (2018).

37. Rummel C, et al. Resected Brain Tissue, Seizure Onset Zone and Quantitative EEG Measures: Towards Prediction of Post-Surgical Seizure Control. PLoS One 10, e0141023 (2015).

38. Schoffelen JM, Gross J. Source connectivity analysis with MEG and EEG. Hum Brain Mapp 30, 18571865 (2009).

39. Junges L, Woldman W, Benjamin OJ, Terry JR. Epilepsy surgery: Evaluating robustness using dynamic network models. Chaos 30, 113106 (2020).

40. Lopes MA, Goodfellow M, Terry JR. A Model-Based Assessment of the Seizure Onset Zone Predictive Power to Inform the Epileptogenic Zone. Front Comput Neurosci 13, 25 (2019).

41. Lopes MA, et al. Computational modelling in source space from scalp EEG to inform presurgical evaluation of epilepsy. Clin Neurophysiol 131, 225-234 (2020).

42. Lopes MA, et al. Elevated Ictal Brain Network Ictogenicity Enables Prediction of Optimal Seizure Control. Front Neuro/ 9,98 (2018).

43. Akaike H. A new look at the statistical model identification. IEEE Trans Automat Contr 19, 716-723 (1974).

44. Schwarz G. Estimating the Dimension of a Model. Ann Stat 6, 461-464 (1978).

45. Gavaret M, Dubarry AS, Carron R, Bartolomei F, Trebuchon A, Benar CG. Simultaneous SEEG-MEGEEG recordings Overcome the SEEG limited spatial sampling. Epilepsy Res 128, 68-72 (2016). 
46. Shigeto $\mathrm{H}$, et al. Feasibility and limitations of magnetoencephalographic detection of epileptic discharges: Simultaneous recording of magnetic fields and electrocorticography. Neurological Research 24, 531-536 (2002).

47. Ding L, Worrell GA, Lagerlund TD, He B. Ictal source analysis: localization and imaging of causal interactions in humans. Neuroimage 34, 575-586 (2007).

48. Hassan M, et al. Identification of Interictal Epileptic Networks from Dense-EEG. Brain Topogr 30, 6076 (2017).

49. Sohrabpour A, Cai Z, Ye S, Brinkmann B, Worrell G, He B. Noninvasive electromagnetic source imaging of spatiotemporally distributed epileptogenic brain sources. Nat Commun 11, 1946 (2020).

50. Khambhati AN, Davis KA, Lucas TH, Litt B, Bassett DS. Virtual Cortical Resection Reveals Push-Pull Network Control Preceding Seizure Evolution. Neuron 91, 1170-1182 (2016).

51. Kini LG, et al. Virtual resection predicts surgical outcome for drug-resistant epilepsy. Brain 142, 38923905 (2019).

52. Rosenow F, Luders H. Presurgical evaluation of epilepsy. Brain 124, 1683-1700 (2001).

53. Lopes MA, et al. An optimal strategy for epilepsy surgery: Disruption of the rich-club? PLoS Comput Biol 13, e1005637 (2017).

54. Pittau F, et al. Mapping epileptic activity: sources or networks for the clinicians? Front Neuro/ 5, 218 (2014).

55. van Mierlo P, Holler Y, Focke NK, Vulliemoz S. Network Perspectives on Epilepsy Using EEG/MEG Source Connectivity. Front Neuro/ 10, 721 (2019).

56. Spencer SS. Neural networks in human epilepsy: evidence of and implications for treatment. Epilepsia 43, 219-227 (2002).

57. Brookes MJ, et al. Measuring functional connectivity using MEG: methodology and comparison with fcMRI. Neuroimage 56, 1082-1104 (2011).

58. Lai M, Demuru M, Hillebrand A, Fraschini M. A comparison between scalp- and source-reconstructed EEG networks. Sci Rep 8, 12269 (2018).

59. Bassett DS, Zurn P, Gold JI. On the nature and use of models in network neuroscience. Nat Rev Neurosci 19, 566-578 (2018).

60. Proix T, Jirsa VK, Bartolomei F, Guye M, Truccolo W. Predicting the spatiotemporal diversity of seizure propagation and termination in human focal epilepsy. Nat Commun 9, 1088 (2018). 


\section{Tables}

Table 1. Patient information, MEG seizures, earliest source localisation solution and outcome. Twelve patients had 36 seizures recorded by MEG. 25/36 seizures reconstructed using ViEEG present distinct morphological features of epileptiform discharges in source space. Six patients achieved seizure-freedom. Nine patients had normal MRI scans while three patients had complex lesions. Early-MSL predicted the EZ in six patients (Patients 1, 3, 4, 5, 10, 11), while early-ESL predicted the EZ in five patients (Patients 2, 6, 8, 9, 12). Ictal discharges were not localisable in one patient (Patient 7). One patient from the original thirteen patients published by Plummer et $\mathrm{al}^{31}$ with normal MRI only had interictal discharges captured by HDEEG and MEG and hence was not included in the present study.

\begin{tabular}{|c|c|c|c|c|c|c|}
\hline Patient & MRI & Histology & $\begin{array}{l}\text { MEG seizures } \\
\text { recorded }\end{array}$ & $\begin{array}{l}\text { MEG seizures } \\
\text { reconstructed }\end{array}$ & $\begin{array}{c}\text { Earliest solution } \\
\text { from ESL and MSL }\end{array}$ & Engel outcome \\
\hline 1 & Normal & CD $1 \mathrm{~A}$ & 6 & 1 & MSL & I (Rare non-disabling seizures) \\
\hline 2 & Normal & CD 1 & 2 & 2 & ESL & I (Seizure-free) \\
\hline 3 & Normal & CD $2 A$ & 7 & 6 & MSL & I (Rare non-disabling seizures) \\
\hline 4 & Normal & Non-specific & 8 & 5 & MSL & III (Fewer disabling seizures) \\
\hline 5 & Normal & $\mathrm{CD} I \mathrm{C}$ & 2 & 1 & MSL & I (Seizure free) \\
\hline 6 & Normal & CD 1 & 1 & 1 & ESL & I (Seizure free) \\
\hline 7 & Normal & $\mathrm{CD} 2 \mathrm{~A}$ & 1 & 1 & Non-localising & I (Seizure free) \\
\hline 8 & Normal & CD 1 & 2 & 2 & ESL & I (Seizure free) \\
\hline 9 & Normal & $\mathrm{CD} 2 \mathrm{~B}$ & 2 & 1 & ESL & II (Rare disabling seizures) \\
\hline 10 & $\begin{array}{l}\text { Multi-lobar } \\
\text { dysplasia }\end{array}$ & Normal & 1 & 1 & MSL & I (Non-disabling seizures) \\
\hline 11 & $\begin{array}{c}\text { Multi-lobar } \\
\text { dysplasia }\end{array}$ & Ischemia & 2 & 2 & MSL & III (Fewer disabling seizures) \\
\hline 12 & Right frontal gliosis & Gliosis & 2 & 2 & ESL & I (Seizure free) \\
\hline
\end{tabular}

\section{Figures}


Simultaneous HDEEG and MEG

Ictal MEG signals

Virtual iEEG (ViEEG)

\section{Gradiometers (204 channels)}

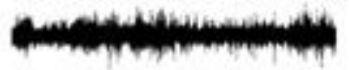

Magnetometers (102 channels)

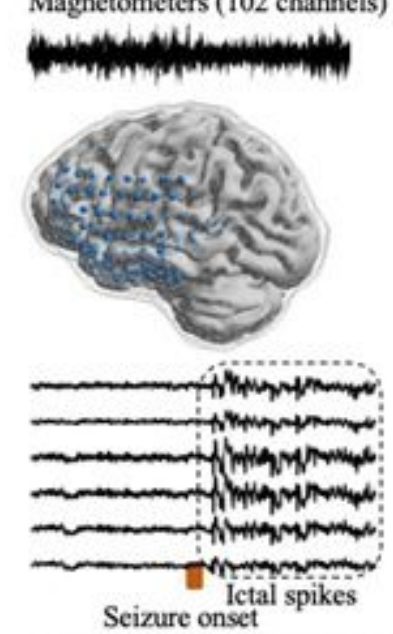

Functional networks and dynamical network models

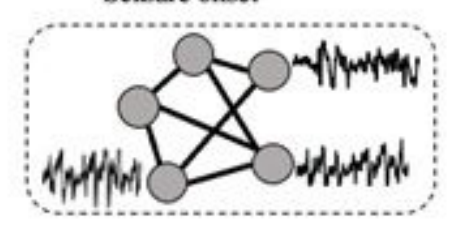

Virtual Ictogenic Zone (VIZ) identified by dynamical models

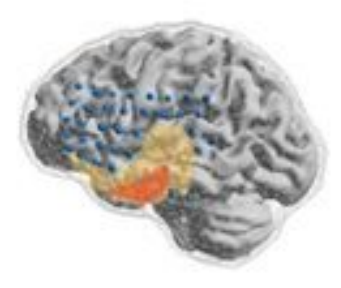

\section{Figure 1}

Workflow of Virtual iEEG (ViEEG) and network model. Simultaneous HDEEG and MEG were acquired from surgical candidates in presurgical evaluation for epilepsy surgery31. Ictal MEG signals from 102-channel magnetometers and 204-channel gradiometers are epoched and pre-processed for source signal reconstruction. ViEEG locations fully contain MSL solutions (early, mid and late phases of averaged ictal discharges) 31 and the entire resection margin. Ictal ViEEG signals are reconstructed using a beamformer technique and a boundary element method (BEM) model generated from individual MRI scans.

Functional networks are constructed using two connectivity methods, amplitude envelope correlation (AEC) and mutual information (MI), and dynamical network models are applied to evaluate how cortical excitability changes when a node is virtually removed from the network. The Virtual Ictogenic Zone (VIZ), identified by a dynamical network approach, consists of a set of nodes that decrease cortical excitability 


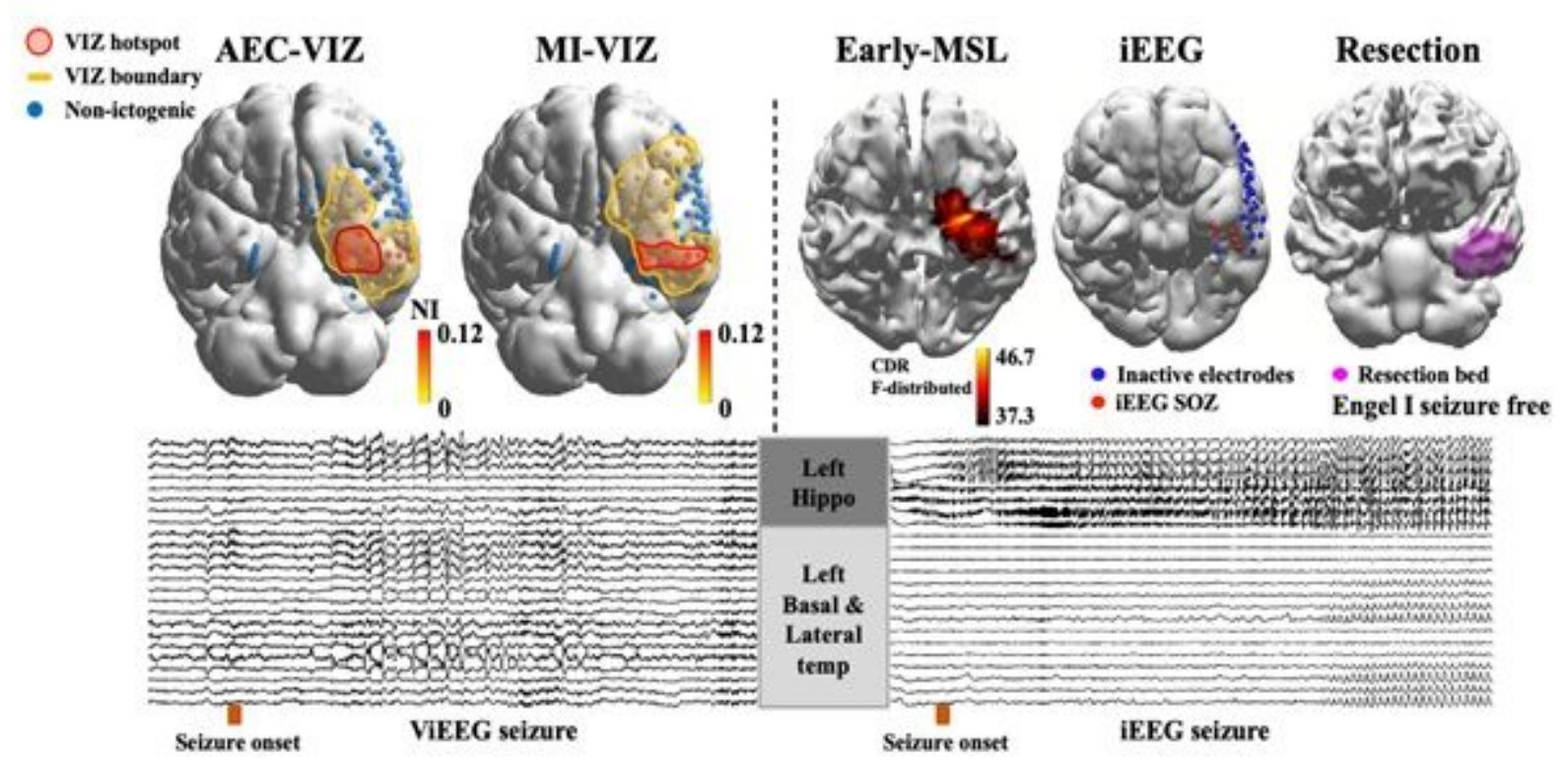

Figure 2

(Patient 5) Morphological and spatial characteristics of ictal ViEEG signals and concordance between VIZ, iEEG SOZ, and early-MSL. Distinct ictal waveforms are seen in the left anterior hippocampal structure and left basal temporal region from a ViEEG seizure aligned in time with seizure onset identified by MEG sensor signals. This is spatially concordant with a seizure that was captured by iEEG after the MEG recording, where the seizure starts from left anterior hippocampus and spreads to the left basal temporal area. AEC-VIZ and MI-VIZ identified by dynamical network models are similar with boundaries containing the iEEG SOZ, resection margin and early-MSL (earliest ictal source localisation solution accounting for $90 \%$ of the signal variance from averaged discharge take-off towards peak) 31 . Both AEC-VIZ and MI-VIZ hotspots (high NI values) point to the left anterior hippocampus, entorhinal cortex and basal temporal structures. The patient has been seizure-free for over two years post-surgery and histology showed cortical dysplasia at the entorhinal cortex. Both AEC-VIZ and MI-VIZ appear to have captured the EZ. 


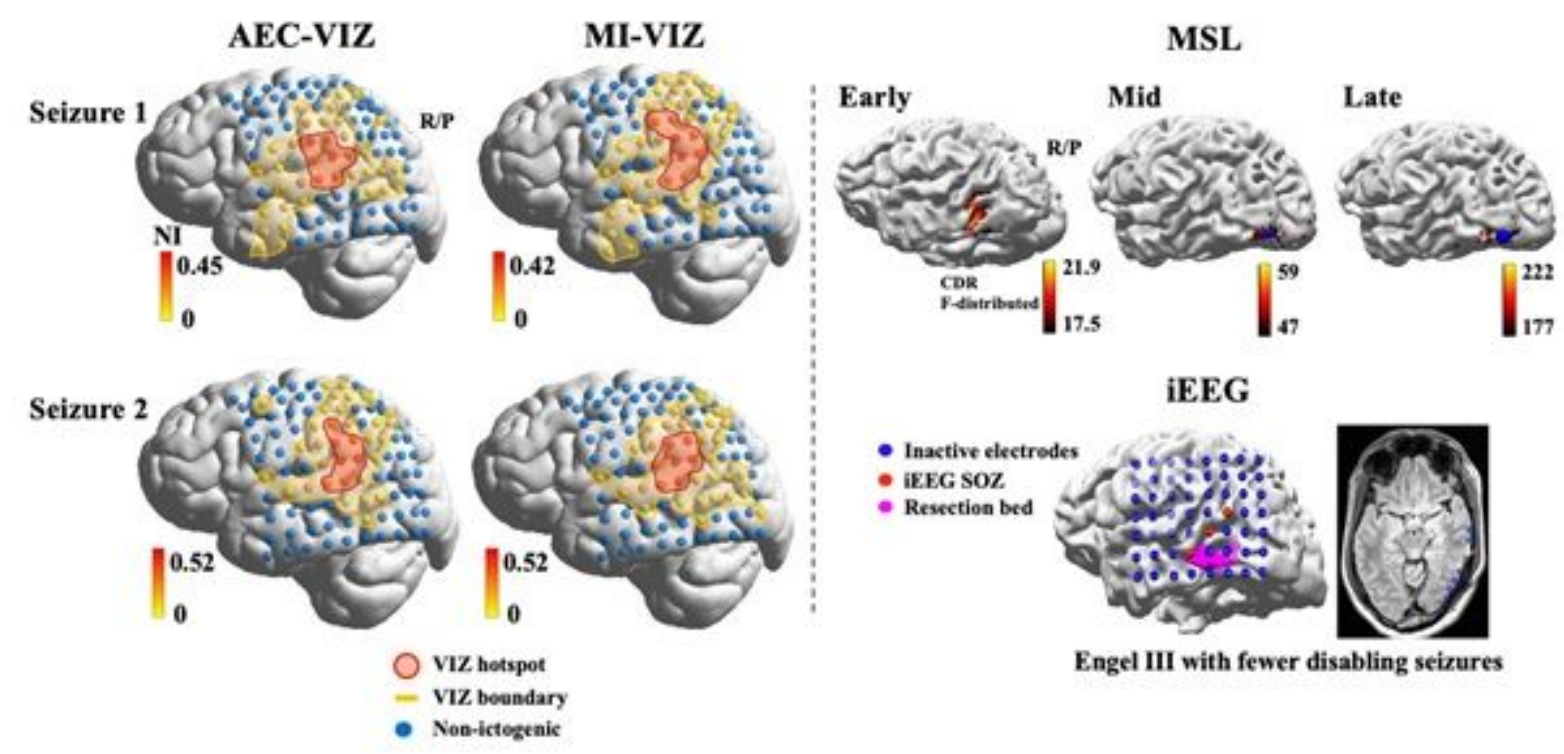

Figure 3

(Patient 11) AEC-VIZ and MI-VIZ hotspot concordance with the earliest source localisation solution (early$\mathrm{MSL}$ ) and resection margin across two seizures suggests an alternative surgical strategy. An alternative surgical plan is suggested by AEC-VIZ and MI-VIZ hotspots (left posterior temporal - parietal junction) from both seizures as opposed to the more discrete left temporo-parieto-occipital junction localisation given by the early-MSL solution. Both VIZ solutions overlapped the iEEG SOZ but lie outside the resection bed (magenta). The patient had a suboptimal surgical outcome (Engel III with fewer disabling seizures) to support the likelihood that the VIZ hotspots are representative of the wider extent of the EZ. 


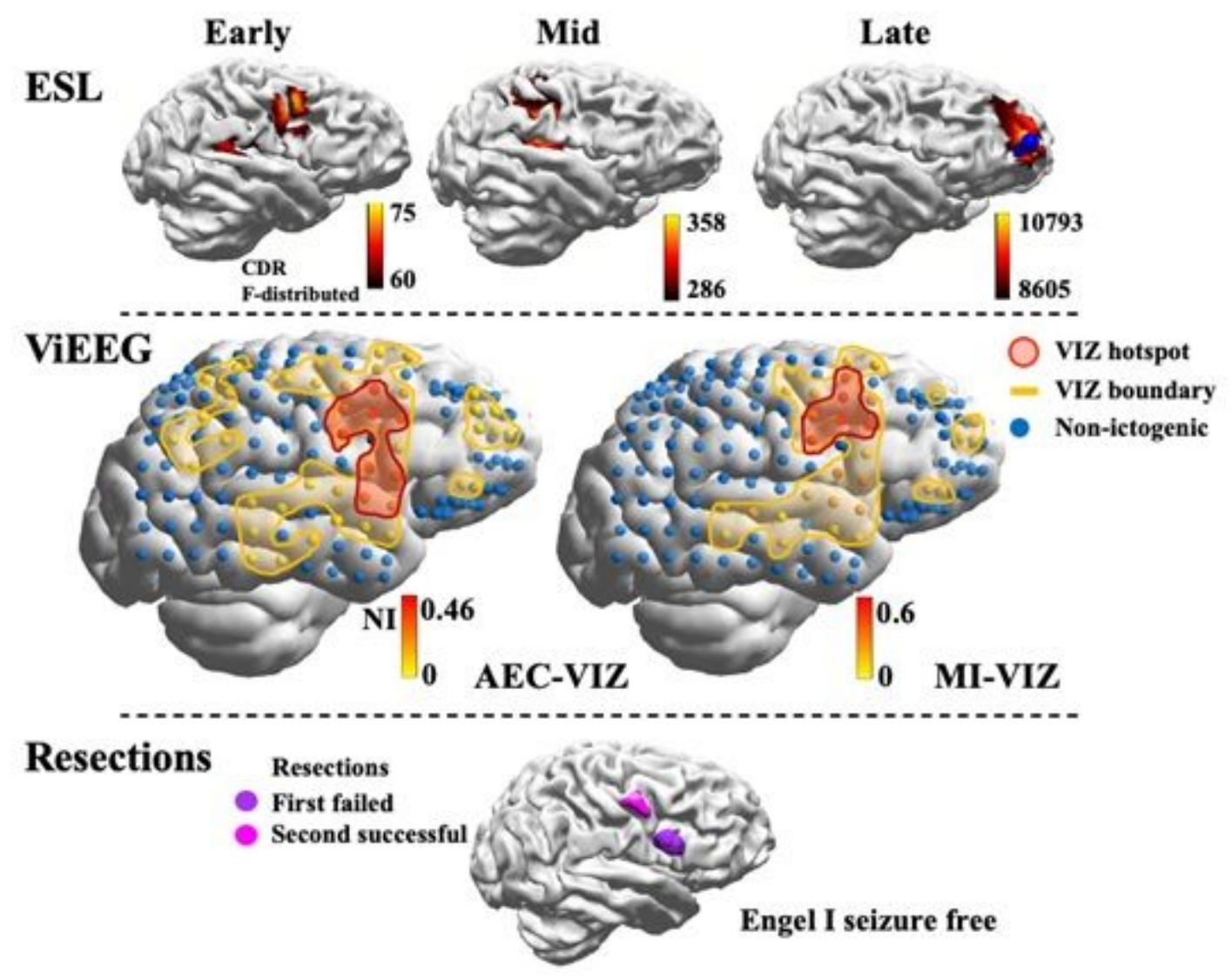

Figure 4

(Patient 6) AEC-VIZ and MI-VIZ hotspot concordance with the earliest source localisation solution (early$E S L$ ) and resection margin. Despite ViEEG being defined by only MSL solutions (only MEG data was used to reconstruct ViEEG signals in this study), dynamical network models suggest AEC-VIZ and MI-VIZ hotspots are concordant with the earliest source localisation solution, which was early-ESL in this case (not early-MSL), and the second resection (magenta). Both hotspots are discordant with MSL (see Supplementary Fig. 10 for early-, mid- and late-MSL solutions). Overlap of AEC-VIZ and MI-VIZ boundaries is also present. Note that this patient had focal motor status epilepticus (left face and hand) and a segment of continuous epileptic discharges is modelled. The second resection (years after the first failed resection with normal histology) led to an Engel I seizure-free outcome at follow-up over two years (histology cortical dysplasia). 

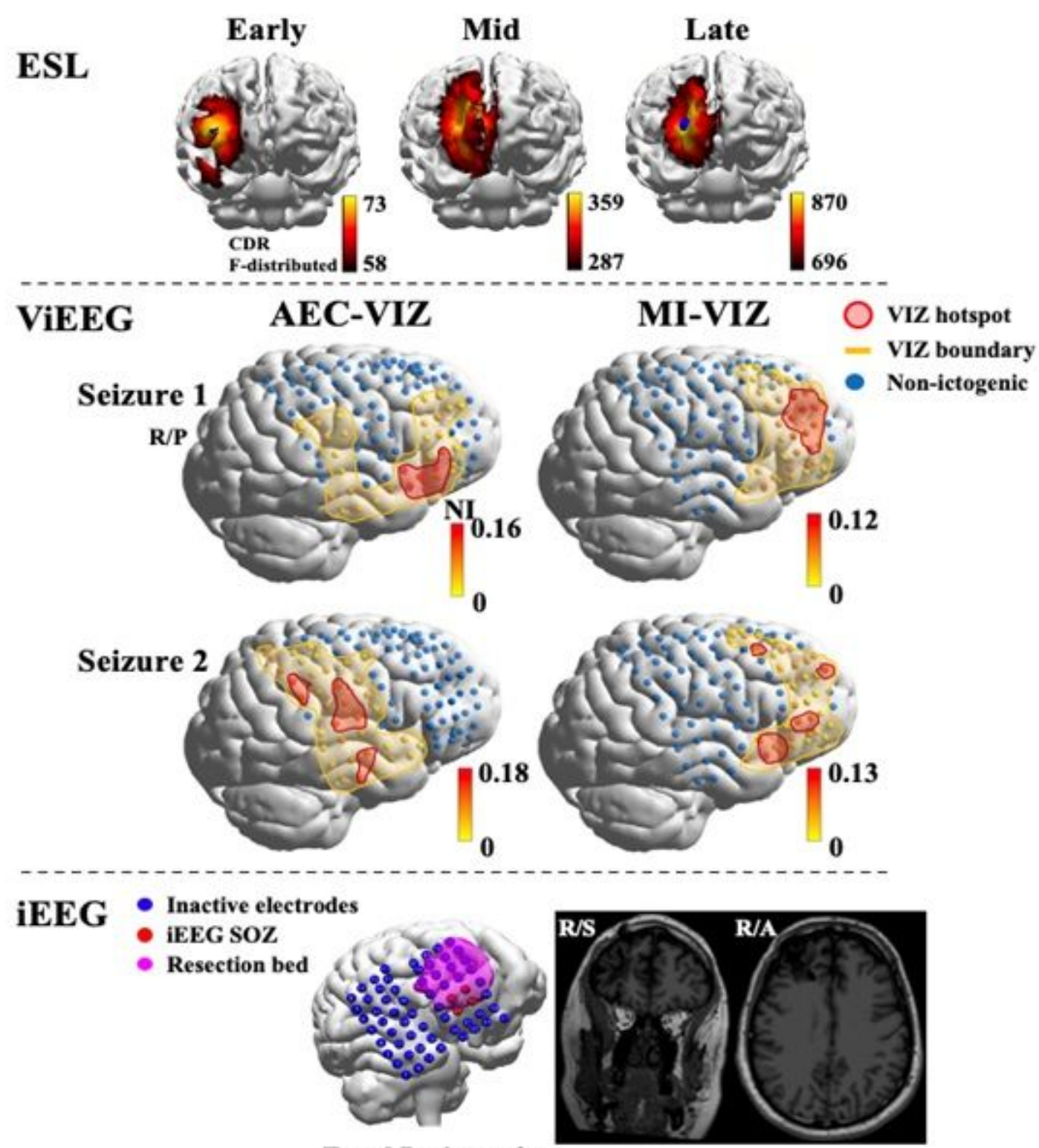

Engel I seizure free

Figure 5

(Patient 12) AEC-VIZ is discordant with MI-VIZ and variability is found across seizures. Dynamical network models identify different hotspots and boundaries between AEC-VIZ and MI-VIZ across two of the recorded seizures. MI-VIZ from seizure 1 gives an extensive boundary in the right frontal lobe with a hotspot that overlaps the resection margin, iEEG SOZ and early-ESL, which is the earliest solution that more accurately predicts the EZ than early-MSL (Supplementary Fig. 16). Boundaries and hotspots for the corresponding $\mathrm{AEC}-\mathrm{VIZ}$ and $\mathrm{MI}-\mathrm{VIZ}$ (seizure 2) hotspots are discordant with early-ESL, resection margin and $\mathrm{IEEG}$ SOZ. The patient is seizure-free over 24 months post-surgery and hence only MI-VIZ from seizure 1 successfully captures the EZ. 
A.

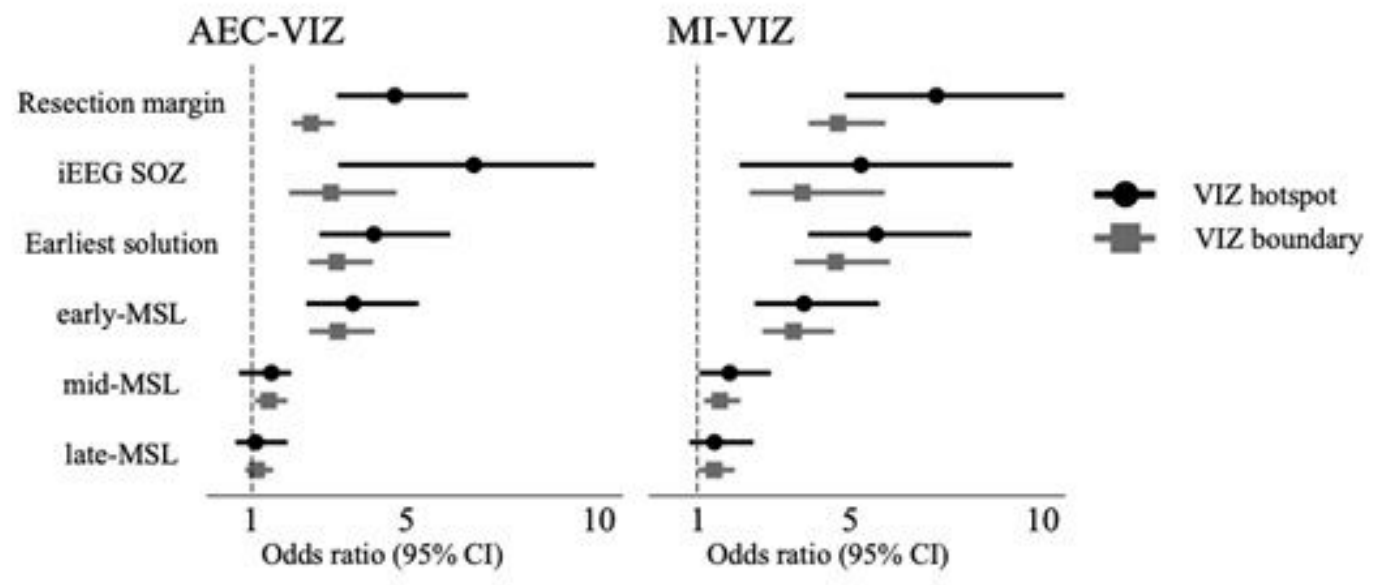

B.

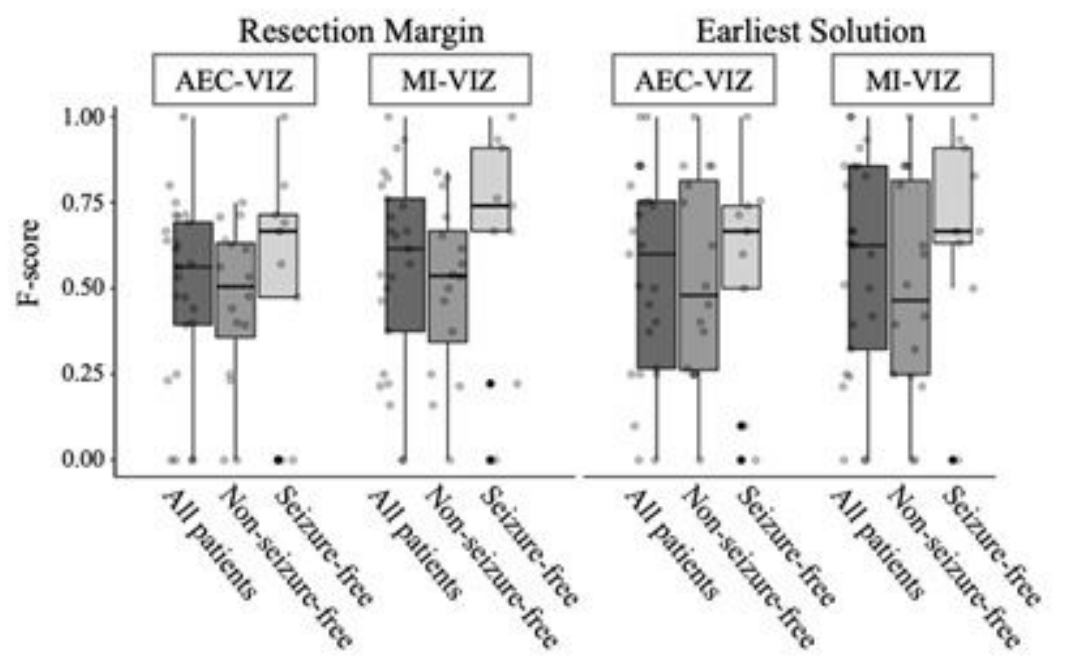

\section{Figure 6}

Statistical findings of AEC and MI-VIZ predicting the resection margin and clinical localisation. A) Odds Ratios (ORs) with 95\% confidence intervals are presented for VIZ hotspot and boundary predicting the resection margin, iEEG SOZ, the earliest solution and MSL solutions (early, mid and late). ORs for AEC-VIZ and $\mathrm{MI}-\mathrm{VIZ}$ hotspots are consistently higher than those of AEC-VIZ and MI-VIZ boundary in predicting the resection margin, iEEG SOZ and the earliest solution. MI-VIZ (hotspot and boundary) have higher ORs than the corresponding AEC-VIZ results when predicting the resection margin. As opposed to AEC-VIZ, MI$\mathrm{VIZ}$ is more likely to predict the resection margin than iEEG SOZ. B) F-scores for AEC-VIZ and MI-VIZ predicting the resection margin and earliest solution are presented in boxplots (horizontal bar, box upper boundary, box lower boundary and dots represent median, first and third quartile and each individual VIZ respectively). MI-VIZ demonstrates higher F-scores (median 0.75) than AEC-VIZ (median 0.68) in predicting the $\mathrm{EZ}$ (i.e., resection margin from seizure-free patients). AEC-VIZ and MI-VIZ do not show a difference between seizure-free and non-seizure-free groups in predicting the earliest solution.

\section{Supplementary Files}


This is a list of supplementary files associated with this preprint. Click to download.

- Supplementarymaterial.pdf 